\title{
KMT-2018-BLG-1990Lb: A Nearby Jovian Planet From A Low-cadence Microlensing Field
}

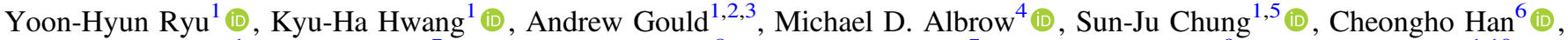 \\ Youn Kil Jung $^{1}$ (D), In-Gu Shin ${ }^{7}$ (D), Yossi Shvartzvald ${ }^{8}$ (iD), Jennifer C. Yee ${ }^{7}$ (D), Weicheng Zang ${ }^{9}$ (iD), Sang-Mok Cha ${ }^{1,10}$, \\ Dong-Jin Kim ${ }^{1}$, Hyoun-Woo Kim ${ }^{1}$, Seung-Lee Kim ${ }^{1,5}$, Chung-Uk Lee ${ }^{1,5}$, Dong-Joo Lee ${ }^{1}$, Yongseok Lee ${ }^{1,10}$, \\ Byeong-Gon Park ${ }^{1,5}$, and Richard W. Pogge ${ }^{3}$ (iD \\ ${ }^{1}$ Korea Astronomy and Space Science Institute, Daejon 34055, Republic of Korea \\ ${ }^{2}$ Max-Planck-Institute for Astronomy, Königstuhl 17, D-69117 Heidelberg, Germany \\ ${ }^{3}$ Department of Astronomy, Ohio State University, 140 W. 18th Avenue, Columbus, OH 43210, USA \\ ${ }^{4}$ University of Canterbury, Department of Physics and Astronomy, Private Bag 4800, Christchurch 8020, New Zealand \\ ${ }^{5}$ Korea University of Science and Technology, Daejeon 34113, Republic of Korea \\ ${ }^{6}$ Department of Physics, Chungbuk National University, Cheongju 28644, Republic of Korea \\ ${ }_{8}^{7}$ Harvard-Smithsonian Center for Astrophysics, 60 Garden Street, Cambridge, MA 02138, USA \\ ${ }^{8}$ IPAC, Mail Code 100-22, Caltech, 1200 E. California Boulevard, Pasadena, CA 91125, USA \\ ${ }^{9}$ Physics Department and Tsinghua Centre for Astrophysics, Tsinghua University, Beijing 100084, People's Republic of China \\ ${ }^{10}$ School of Space Research, Kyung Hee University, Yongin, Kyeonggi 17104, Republic of Korea \\ Received 2019 February 21; revised 2019 August 7; accepted 2019 August 8; published 2019 September 20
}

\begin{abstract}
We report the discovery of KMT-2018-BLG-1990Lb, a Jovian planet ( $\left.m_{p}=0.57_{-0.25}^{+0.79} M_{J}\right)$ orbiting a late M dwarf $\left(M=0.14_{-0.06}^{+0.20} M_{\odot}\right)$ at a distance of $\left(D_{L}=1.23_{-0.43}^{+1.06} \mathrm{kpc}\right)$, and projected at $2.6 \pm 0.6$ times the snow line distance, i.e., $a_{\text {snow }} \equiv 2.7 \mathrm{au}\left(M / M_{\odot}\right)$. This is the second Jovian planet discovered by the Korea Microlensing Telescope Network (KMTNet) in its low-cadence $\left(0.4 \mathrm{hr}^{-1}\right)$ fields, demonstrating that this population can be probed based on relatively low-cadence survey-only microlensing data. However, while many Jovian-planet microlensing events will not have caustic crossings (e.g., the previous case, KMT-2016-BLG-1397) and some that do will be well characterized by low-cadence survey observations (e.g., the current case), in general higher-cadence follow-up observations of the caustic crossings are preferable. Such follow-up observations require microlensing alerts, which KMTNet now provides.
\end{abstract}

Key words: gravitational lensing: micro

\section{Introduction}

For many years (beginning with the second microlensing planet OGLE-2005-BLG-071Lb; Udalski et al. 2005), most microlensing planets were discovered by intensive follow-up observations of microlensing events that were alerted by wide-field surveys. Gould \& Loeb (1992) had advocated such a strategy because microlensing events, which have typical Einstein timescales of $t_{\mathrm{E}} \sim 20$ days, can be discovered even in very low-cadence surveys $\Gamma \lesssim 1$ day $^{-1}$, whereas the fleeting appearance of planetary anomalies generally requires much higher cadence to detect and, more critically, to reasonably characterize the planetary anomalies. In their scheme, the microlensing surveys could cover very broad areas from even a single site, while narrow-angle follow-up observations could be carried out around the clock at much higher cadence on a handful of favorable targets.

The Korea Microlensing Telescope Network (KMTNet; Kim et al. 2016) monitors about $97 \mathrm{deg}^{2}$ toward the Galactic bulge from three $1.6 \mathrm{~m}$ telescopes that are equipped with $4 \mathrm{deg}^{2}$ cameras, in Chile (KMTC), South Africa (KMTS), and Australia (KMTA). Its primary goal is to find exoplanets via the anomalies that these generate in microlensing events through round-theclock monitoring. By continuously monitoring a broad area at relatively high-cadence from its three sites, KMTNet aimed to simultaneously find microlensing events and find and characterize the planetary anomalies within them, without any follow-up observations (and hence without the necessity of microlensing alerts). In 2015, its first (commissioning) year of observation, KMTNet narrowly focused on this strategy (Kim et al. 2018a). It observed only four fields, allowing a very high cadence of $\Gamma=6 \mathrm{hr}^{-1}$, which as we will discuss immediately below, was sufficient to detect planets down to about one Earth mass. ${ }^{11}$

However, beginning in 2016, KMTNet developed a radically different strategy, with a range of cadences, $\Gamma=(4,1$, $0.4,0.2) \mathrm{hr}^{-1}$, covering areas ${ }^{12}$ of $(12,29,44,12) \mathrm{deg}^{2}$, respectively. See Figure 12 of Kim et al. (2018a). This change was motivated by a variety of goals, including support for Spitzer microlensing (Gould et al. 2013, 2014, 2015a, 2015b, 2016, 2018), which is an intrinsically wide-field experiment. However, a major goal was simply to find more planets over a much broader area.

For a typical microlensing event, the half-duration of a planetary perturbation is roughly the planetary Einstein time, although this is not the case for "Hollywood" events (Gould 1997) such as OGLE-2005-BLG-390 (Beaulieu et al. 2006)

$$
\begin{aligned}
t_{\mathrm{E}, p}= & \frac{\theta_{\mathrm{E}, p}}{\mu_{\mathrm{rel}}} \simeq 1.25 \mathrm{hr}\left(\frac{m_{p}}{M_{\oplus}}\right)^{1 / 2}\left(\frac{\pi_{\mathrm{rel}}}{0.02 \mathrm{mas}}\right)^{1 / 2} \\
& \times\left(\frac{\mu_{\mathrm{rel}}}{5 \mathrm{mas} \mathrm{yr}^{-1}}\right)^{-1},
\end{aligned}
$$

\footnotetext{
11 In fact, in 2015 KMTNet augmented this strategy with very low-cadence (1-2 per day per observatory) observations of 15 other fields in support of Spitzer microlensing. However, because these constituted $\lesssim 10 \%$ of all observations, they did not significantly impact the basic strategy.

${ }^{12}$ This breakdown of cadences is somewhat oversimplified. See Kim et al. (2018b) for a more detailed summary.
} 
where

$$
\begin{aligned}
& \theta_{\mathrm{E}, p} \equiv \sqrt{\frac{q}{1+q}} \theta_{\mathrm{E}} ; \quad \theta_{\mathrm{E}} \equiv \sqrt{\kappa M \pi_{\mathrm{rel}}} \\
& \kappa \equiv \frac{4 G}{c^{2} \mathrm{au}} \simeq 8.14 \frac{\mathrm{mas}}{M_{\odot}}
\end{aligned}
$$

$q \equiv m_{p} / M$ is the planet-host mass ratio, $\theta_{\mathrm{E}}$ is the Einstein radius, and $\pi_{\text {rel }}$ and $\boldsymbol{\mu}_{\text {rel }}$ are, respectively, the lens-source relative parallax and proper motion. Thus, assuming that about 10 data points are needed over the full anomaly, the adopted cadences of $\Gamma=(4,1,0.4,0.2) \mathrm{hr}^{-1}$ should be sufficient to detect planets of mass $m_{p} \sim(1,16,100,400) M_{\oplus}$, corresponding to Earth, Neptune, Saturn and Jupiter mass planets. See also Kim et al. (2018c) and Figure 14 from Henderson et al. (2014). In effect, the revised strategy sacrificed some sensitivity to Earth-mass planets over $4 \mathrm{deg}^{2}$ in order to expand the total area by a factor 6 , including a 2.5 fold increase in the area sensitive to Neptunes.

Here we report on KMT-2018-BLG-1990Lb, a Jovian-mass planet lying in field BLG38, which is monitored by KMTNet at $\Gamma=0.4 \mathrm{hr}^{-1}$ and at a position that was not monitored by other surveys. It is the second KMT-only Jovian planet from a field with this cadence, the first being KMT-2016-BLG-1397Lb from BLG31 (Zang et al. 2018). As such, it demonstrates the viability of a wide-field survey for gas giant planets in accord with the KMTNet strategy.

\section{Observations}

KMT-2018-BLG-1990 is at (R.A., decl.) $=(17: 53: 44.49$, $-22: 09: 09.14)$, corresponding to $(l, b)=(6.77,1.91)$, i.e., well out along the near side of the Galactic bar. As mentioned in Section 1, it lies in KMTNet field BLG38, which has a nominal cadence of $\Gamma=0.4 \mathrm{hr}^{-1}$. However, from the start of the season through 2018 June 25, KMTNet followed a modified survey strategy in which BLG38 continued to be observed at $\Gamma=0.4 \mathrm{hr}^{-1}$ from KMTC, but was observed at $\Gamma=0.3 \mathrm{hr}^{-1}$ from KMTS and KMTA. This period contained virtually the whole of the event.

The great majority of observations were carried out in the $I$ band, but about $9 \%$ were in the $V$ band. The primary purpose of the latter was to determine the source color. All reductions for this analysis were conducted using variants of image subtraction (Alard \& Lupton 1998). In particular, a variant of the Woźniak (2000) difference image analysis (DIA) code was used for pipeline reductions of the 500 million light curves that were searched for microlensing events. Then pySIS (Albrow et al. 2009) pipeline re-reductions were carried out to further check whether the event is indeed microlensing, and finally tender loving care (TLC) pySIS re-reductions were used to derive parameters. A separate package, pyDIA (Albrow 2017), was used to construct the color-magnitude diagram (CMD).

The event was discovered by applying the KMTNet eventfinder algorithm (Kim et al. 2018a) to the 2018 DIA light curves. Very briefly, a machine review of these light curves chose this event (among 100,130 candidates) for human review. The DIA light curve (which can be accessed at http://kmtnet.kasi.re.kr/ ulens/) is easily and securely identified as microlensing, but actually displays no clear signature of an anomaly. The anomaly was discovered after running pipeline pySIS on the BLG38 candidates in order to conduct a final review of these candidates.
The pipeline pySIS reduction (also accessible at http://kmtnet. kasi.re.kr/ulens/) shows a double-horned anomaly, characteristic of a planetary or binary caustic crossing, which was immediately investigated and revealed to be planetary. By chance, this was the first 2018 event-finder microlensing-event candidate that was examined manually.

\section{Analysis}

Figure 1 shows the KMT-2018-BLG-1990 data together with the best-fit model. The light curve shows a roughly $65 \mathrm{hr}$ anomaly just before the peak of an otherwise normal Paczyński (1986) point-lens light curve. According to the naive scaling given in Section 1, this should correspond to an $m_{p} \sim 10^{3} M_{\oplus}$ companion. It should be noted that a broadly similar morphology can be generated when a source transits the relatively small Chang-Refsdal caustic of a wide $(s \gg 1)$ or close $(s \ll 1)$ binary with comparable mass components, followed by a cusp approach. In that case, however, one would expect that the peak would be offset from the center of the light curve as defined by the wings. Nevertheless, this example serves as a caution that a thorough examination of parameter space should be undertaken, even when the caustic geometry appears obvious.

We proceed by a standard search for solutions defined by seven nonlinear parameters: $\left(t_{0}, u_{0}, t_{\mathrm{E}}, s, q, \alpha, \rho\right)$. The first three are the Paczyński (1986) parameters of the underlying point-lens events: time of closest approach, impact parameter in units of $\theta_{\mathrm{E}}$, Einstein timescale $t_{\mathrm{E}}=\theta_{\mathrm{E}} / \mu_{\text {rel }}$. The next three define the binary geometry: separation in units of $\theta_{\mathrm{E}}$, mass ratio, orientation of binary axis relative to $\boldsymbol{\mu}_{\mathrm{rel}}$. The last, $\rho=\theta_{*} / \theta_{\mathrm{E}}$, is the source radius normalized to the Einstein radius where $\theta_{*}$ is the angular radius of the source. In addition, there are two flux parameters for each observatory $i$, i.e., the source flux $f_{s, i}$ and the blend flux $f_{b, i}$, so that the model flux is given by $F_{i}(t)=f_{s, i} A(t)+f_{b, i}$, where $A(t)$ is the model magnification.

We first conduct a grid search, holding $(s, q)$ fixed on a grid of values and allowing all other parameters to vary in a Monte Carlo Markov chain (MCMC). For the three Paczyński (1986) parameters, we seed the chains with the results from a pointlens fit (with the anomaly removed). For $\alpha$, we seed with six values taken uniformly around the unit circle. Finally, we seed the normalized source radius with $\rho=10^{-3}$. The flux parameters are determined by a linear fit to the data for each trial model. We find that there are two solutions, which are related by the so-called close-wide degeneracy, which approximately takes $s \rightarrow s^{-1}$. In fact, this degeneracy was originally derived in the limits $s \gg 1$ or $s \ll 1$ (Griest \& Safizadeh 1998), but sometimes holds when $s \sim 1$ as well.

We then conduct a refined search on each of these two solutions by allowing all seven geometric parameters to vary in the MCMC. The results are shown in Table 1. Note that, in contrast to the limiting cases from which this degeneracy is derived, the $s \rightarrow s^{-1}$ transformation does not preserve the value of $q$. Instead, $q(s<1) \sim 3.7 \times 10^{-3}$, while $q(s>1) \sim 6.2 \times 10^{-3}$.

\subsection{Microlens Parallax: $\pi_{\mathrm{E}}$}

Next, we consider the microlens parallax effect. A combination of two facts strongly suggests that this effect will be measurable, or at least strongly constrained. First, the timescale is relatively long, $t_{\mathrm{E}} \sim 45$ days, implying that Earth's velocity projected on the sky changes by $\Delta v \sim 35 \mathrm{~km} \mathrm{~s}^{-1}$ 

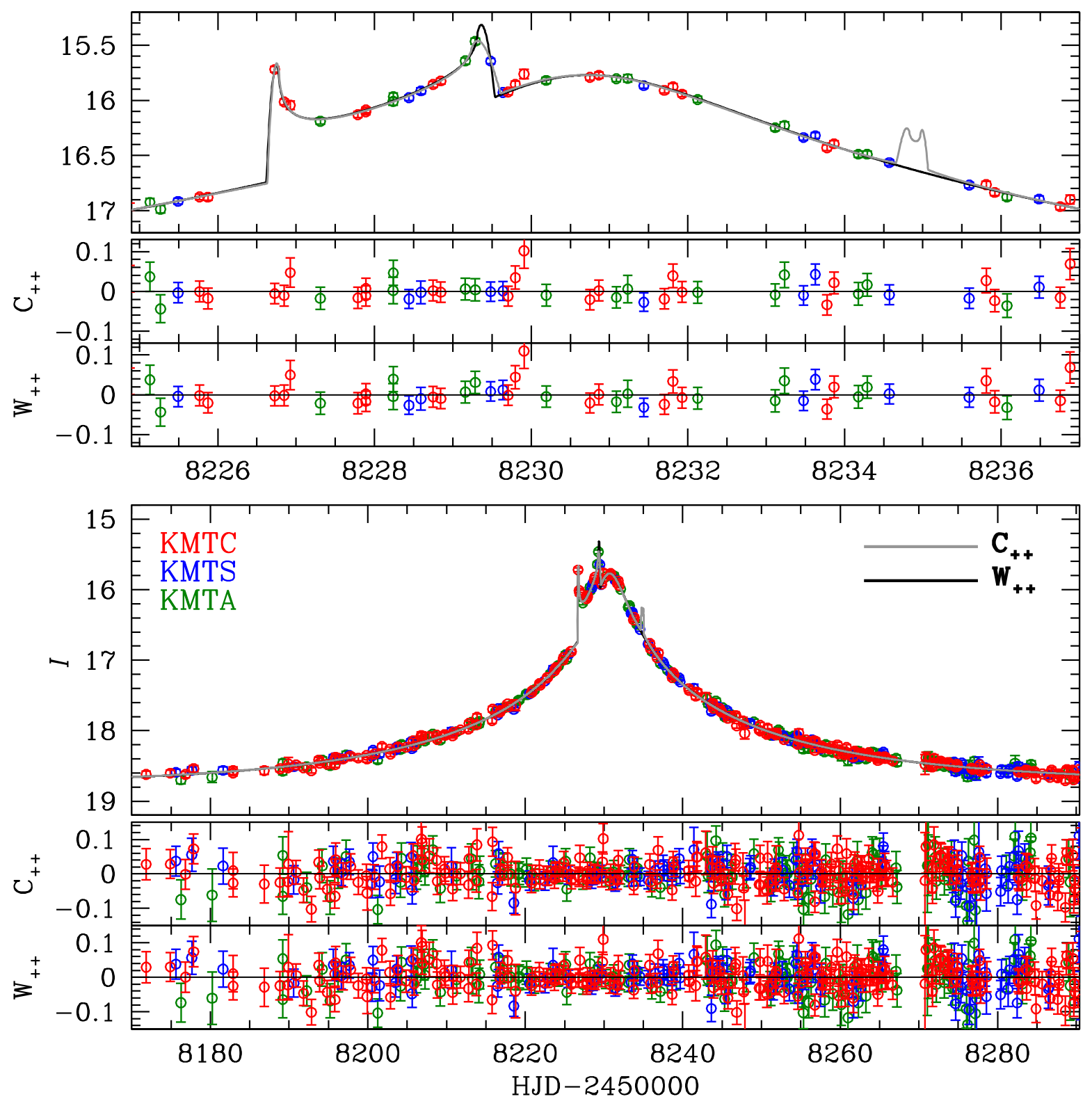

Figure 1. Light curve of KMT-2018-BLG-1990 with data from the three KMTNet observatories. The curves show the $C_{++}$and $W_{++}$models from Tables 2 and 3 , but the other six degenerate models in these tables look extremely similar.

Table 1

Standard Models

\begin{tabular}{lrr}
\hline \hline & \multicolumn{1}{c}{ Close } & \multicolumn{1}{c}{ Wide } \\
\hline$\chi^{2} /$ dof & $786.061 / 749$ & $788.832 / 749$ \\
$t_{0}\left(\mathrm{HJD}^{\prime}\right)$ & $8230.495 \pm 0.019$ & $8230.370 \pm 0.021$ \\
$u_{0}$ & $0.044 \pm 0.001$ & $0.039 \pm 0.001$ \\
$t_{\mathrm{E}}($ days $)$ & $43.929 \pm 0.754$ & $45.299 \pm 0.984$ \\
$s$ & $0.968 \pm 0.001$ & $1.146 \pm 0.004$ \\
$q\left(10^{-3}\right)$ & $3.723 \pm 0.167$ & $6.229 \pm 0.315$ \\
$\alpha(\mathrm{rad})$ & $2.559 \pm 0.007$ & $2.482 \pm 0.007$ \\
$\rho\left(10^{-3}\right)$ & $1.280 \pm 0.144$ & $1.260 \pm 0.155$ \\
$f_{S}$ & $0.356 \pm 0.008$ & $0.344 \pm 0.010$ \\
$f_{B}$ & $0.147 \pm 0.007$ & $0.158 \pm 0.008$ \\
$t_{*}($ days $)$ & $0.056 \pm 0.006$ & $0.057 \pm 0.007$ \\
\hline
\end{tabular}

Note. The zero point for fluxes is 18 , e.g., $I_{S}=18-2.5 \log \left(f_{S}\right) . t_{*} \equiv \rho t_{\mathrm{E}}$ is a derived quantity and is not separately fitted. during the $\sim 2 t_{\mathrm{E}}$ time interval when the source is significantly magnified.

Second, as we will show in Section 4.1, $\theta_{*} \sim 1.2 \mu$ as. Combining this with the measured value of $\rho \sim 1.2 \times 10^{-3}$ implies $\theta_{\mathrm{E}}=\theta_{*} / \rho \simeq 1.0$ mas. The microlens parallax vector is given by

$$
\boldsymbol{\pi}_{\mathrm{E}} \equiv \frac{\pi_{\mathrm{rel}}}{\theta_{\mathrm{E}}} \frac{\boldsymbol{\mu}_{\mathrm{rel}}}{\mu_{\mathrm{rel}}}
$$

which immediately yields (Gould 1992, 2000),

$$
\pi_{\mathrm{rel}}=\theta_{\mathrm{E}} \pi_{\mathrm{E}} ; \quad M=\frac{\theta_{\mathrm{E}}}{\kappa \pi_{\mathrm{E}}} .
$$

Hence, $\theta_{\mathrm{E}} \simeq 1.0$ mas implies $M \lesssim 1 M_{\odot}$ and $\pi_{\text {rel }} \lesssim 0.13$ mas. Otherwise the lens would be easily visible in the blended light. 


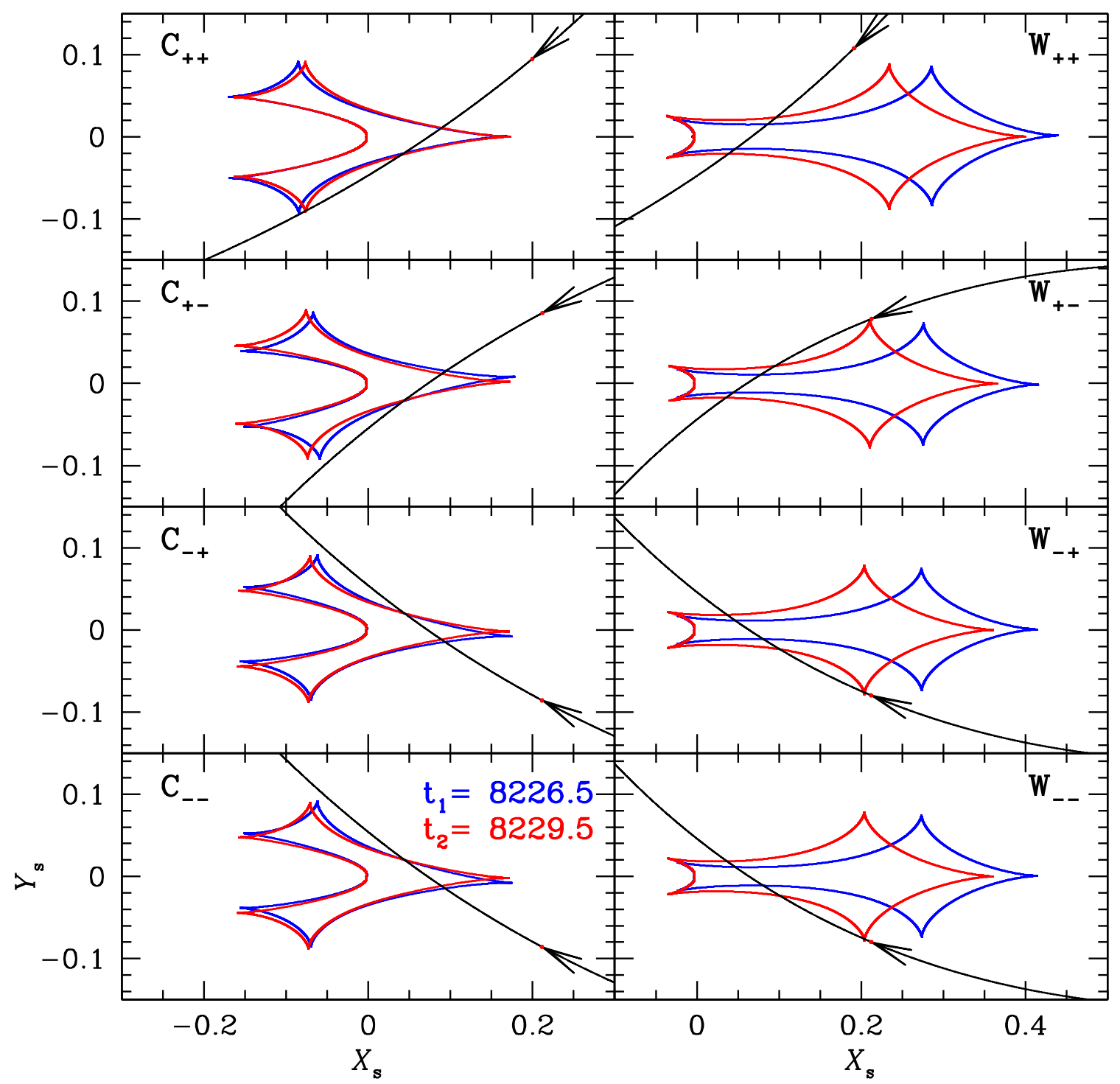

Figure 2. Caustic geometries for each of the eight solutions shown in Tables 2 and 3 . The red and blue closed curves show the caustics at the two designated epochs, while the black curves show the source trajectory relative to these structures.

Then, from the limit on $\pi_{\text {rel }}$, we can also place limits on the amplitude of the lens-source projected velocity $\tilde{\mathbf{v}} \equiv$ $\left(\mathrm{au} / t_{\mathrm{E}}\right) \pi_{\mathrm{E}} / \pi_{\mathrm{E}}^{2}$

$$
\tilde{v}=\frac{\mathrm{au}}{t_{\mathrm{E}} \pi_{\mathrm{E}}}=\frac{\mathrm{au}}{\pi_{\mathrm{rel}}} \frac{\theta_{\mathrm{E}}}{t_{\mathrm{E}}} \lesssim 290 \mathrm{~km} \mathrm{~s}^{-1}
$$

That is, during the event, the lens-source motion as observed from Earth changes fractionally by at least $\Delta v / \tilde{v} \gtrsim 12 \%$. We introduce two additional parameters $\left(\pi_{\mathrm{E}, N}, \pi_{\mathrm{E}, E}\right)$, which are the components of $\pi_{\mathrm{E}}$ in the Equatorial coordinate system. Because the effects of Earth's orbital motion can be correlated with the effects of lens orbital motion, it is essential to simultaneously consider the latter, which we parameterize by $(d s / d t, d \alpha / d t)$, the instantaneous changes in the separation and orientation of the two components at $t_{0}$. In fact, we find that the orbital parameters are only weakly constrained. We therefore restrict the MCMC trials by the condition $\beta<0.8$, where $\beta$ is the ratio of projected kinetic to potential energy

$$
\begin{aligned}
& \beta=\left|\frac{\mathrm{KE}}{\mathrm{PE}}\right|_{\perp}=\frac{\kappa M_{\odot} \mathrm{yr}^{2}}{8 \pi^{2}} \frac{\pi_{\mathrm{E}}}{\theta_{\mathrm{E}}} \gamma^{2}\left(\frac{s}{\pi_{\mathrm{E}}+\pi_{s} / \theta_{\mathrm{E}}}\right)^{3} ; \\
& \gamma \equiv\left(\frac{d s / d t}{s}, \frac{d \alpha}{d t}\right),
\end{aligned}
$$

where we adopt $\pi_{s}=0.14$ mas for the source parallax.

As usual, we must consider the ecliptic degeneracy, which takes $\left(u_{0}, \alpha, \pi_{\mathrm{E}, \perp}, d \alpha / d t\right) \rightarrow-\left(u_{0}, \alpha, \pi_{\mathrm{E}, \perp}, d \alpha / d t\right)$ (Skowron et al. 2011), where $\pi_{\mathrm{E}, \perp}$ is the component of $\pi_{\mathrm{E}}$ that is perpendicular to the direction of Earth's acceleration (projected on the sky) at $t_{0}$. Indeed, because the event is very close to the ecliptic, $\beta_{\text {ecliptic }}=+1^{\circ} .3$, we expect this degeneracy to be very severe: it is exact in the limit $\beta_{\text {ecliptic }} \rightarrow 0$.

We find that for each of the four near-degenerate combinations, $[(s<1),(s>1)] \times\left[\left(u_{0}<0\right),\left(u_{0}>0\right)\right]$, there are two local minima, which are characterized by different values of $\pi_{\mathrm{E}, N}$. Such a discrete degeneracy is predicted by the jerk-parallax 
Table 2

Parallax+orbital Motion Models for the Close-separation

\begin{tabular}{|c|c|c|c|c|}
\hline \multirow[b]{2}{*}{ Parameters } & \multicolumn{2}{|c|}{$u_{0}>0$} & \multicolumn{2}{|c|}{$\bar{~} u_{0}<0$} \\
\hline & $\pi_{\mathrm{E}, N}>0$ & $\pi_{\mathrm{E}, N}<0$ & $\pi_{\mathrm{E}, N}>0$ & $\pi_{\mathrm{E}, N}<0$ \\
\hline$\overline{\chi^{2} / \text { dof }}$ & $743.899 / 745$ & $742.074 / 745$ & $742.013 / 745$ & $743.720 / 745$ \\
\hline$t_{0}\left(\mathrm{HJD}^{\prime}\right)$ & $8230.449 \pm 0.024$ & $8230.466 \pm 0.024$ & $8230.465 \pm 0.024$ & $8230.448 \pm 0.023$ \\
\hline$u_{0}$ & $0.040 \pm 0.002$ & $0.043 \pm 0.002$ & $-0.043 \pm 0.002$ & $-0.040 \pm 0.002$ \\
\hline$t_{\mathrm{E}}$ (days) & $46.484 \pm 1.945$ & $45.372 \pm 1.760$ & $45.908 \pm 2.228$ & $46.223 \pm 2.260$ \\
\hline$s$ & $0.963 \pm 0.003$ & $0.960 \pm 0.007$ & $0.963 \pm 0.007$ & $0.963 \pm 0.005$ \\
\hline$q\left(10^{-3}\right)$ & $3.748 \pm 0.253$ & $3.648 \pm 0.370$ & $3.529 \pm 0.359$ & $3.774 \pm 0.303$ \\
\hline$\alpha(\mathrm{rad})$ & $2.584 \pm 0.018$ & $2.488 \pm 0.017$ & $3.794 \pm 0.021$ & $3.699 \pm 0.020$ \\
\hline$\rho\left(10^{-3}\right)$ & $1.187 \pm 0.162$ & $1.423 \pm 0.150$ & $1.388 \pm 0.157$ & $1.228 \pm 0.159$ \\
\hline$\pi_{\mathrm{E}, N}$ & $1.147 \pm 0.349$ & $-1.428 \pm 0.273$ & $1.386 \pm 0.262$ & $-1.122 \pm 0.355$ \\
\hline$\pi_{\mathrm{E}, E}$ & $0.140 \pm 0.031$ & $0.211 \pm 0.046$ & $0.198 \pm 0.049$ & $0.146 \pm 0.034$ \\
\hline$d s / d t\left(\mathrm{yr}^{-1}\right)$ & $0.503 \pm 0.232$ & $-0.695 \pm 0.574$ & $-0.359 \pm 0.539$ & $0.509 \pm 0.548$ \\
\hline$d \alpha / d t\left(\mathrm{yr}^{-1}\right)$ & $-0.302 \pm 1.197$ & $-4.136 \pm 1.764$ & $4.139 \pm 2.294$ & $0.163 \pm 1.244$ \\
\hline$f_{S}$ & $0.325 \pm 0.016$ & $0.353 \pm 0.015$ & $0.347 \pm 0.018$ & $0.328 \pm 0.017$ \\
\hline$f_{B}$ & $0.176 \pm 0.016$ & $0.151 \pm 0.014$ & $0.154 \pm 0.017$ & $0.175 \pm 0.017$ \\
\hline$t_{*}$ (days) & $0.055 \pm 0.007$ & $0.065 \pm 0.007$ & $0.064 \pm 0.007$ & $0.057 \pm 0.007$ \\
\hline$\beta$ & $0.018 \pm 0.111$ & $0.653 \pm 0.217$ & $0.666 \pm 0.201$ & $0.016 \pm 0.154$ \\
\hline
\end{tabular}

Note. The parameter $\beta$ is restricted to $\beta<0.8$. The parameters $\beta$ (Equation (6)) and $t_{*} \equiv \rho t_{\mathrm{E}}$ are derived quantities and are not separately fitted.

Table 3

Parallax+orbital Motion Models for the Wide-separation

\begin{tabular}{|c|c|c|c|c|}
\hline \multirow[b]{2}{*}{ Parameters } & \multicolumn{2}{|c|}{$u_{0}>0$} & \multicolumn{2}{|c|}{$u_{0}<0$} \\
\hline & $\pi_{\mathrm{E}, N}>0$ & $\pi_{\mathrm{E}, N}<0$ & $\pi_{\mathrm{E}, N}>0$ & $\pi_{\mathrm{E}, N}<0$ \\
\hline$\overline{\chi^{2} / \text { dof }}$ & $746.620 / 745$ & $743.078 / 745$ & $742.497 / 745$ & $745.785 / 745$ \\
\hline$u_{0}$ & $0.039 \pm 0.002$ & $0.035 \pm 0.002$ & $-0.037 \pm 0.002$ & $-0.039 \pm 0.002$ \\
\hline$t_{\mathrm{E}}($ days $)$ & $45.646 \pm 1.833$ & $53.380 \pm 2.721$ & $51.389 \pm 3.152$ & $46.228 \pm 1.870$ \\
\hline$s$ & $1.115 \pm 0.012$ & $1.099 \pm 0.009$ & $1.095 \pm 0.010$ & $1.118 \pm 0.009$ \\
\hline$\rho\left(10^{-3}\right)$ & $1.223 \pm 0.158$ & $1.175 \pm 0.140$ & $1.246 \pm 0.155$ & $1.336 \pm 0.160$ \\
\hline$\pi_{\mathrm{E}, N}$ & $1.400 \pm 0.287$ & $-1.649 \pm 0.208$ & $1.637 \pm 0.252$ & $-1.521 \pm 0.370$ \\
\hline$\pi_{\mathrm{E}, E}$ & $0.075 \pm 0.047$ & $-0.003 \pm 0.038$ & $-0.002 \pm 0.043$ & $0.056 \pm 0.034$ \\
\hline$d s / d t\left(\mathrm{yr}^{-1}\right)$ & $-3.580 \pm 1.249$ & $-4.464 \pm 0.727$ & $-4.762 \pm 0.098$ & $-3.429 \pm 0.855$ \\
\hline$d \alpha / d t\left(\mathrm{yr}^{-1}\right)$ & $-0.367 \pm 1.397$ & $0.288 \pm 0.665$ & $-0.156 \pm 1.269$ & $-0.033 \pm 0.348$ \\
\hline$f_{S}$ & $0.334 \pm 0.016$ & $0.294 \pm 0.017$ & $0.307 \pm 0.020$ & $0.329 \pm 0.017$ \\
\hline
\end{tabular}

Note. The parameter $\beta$ is restricted to $\beta<0.8$. The parameters $\beta$ (Equation (6)) and $t_{*} \equiv \rho t_{\mathrm{E}}$ are derived quantities and are not separately fitted.

degeneracy (Gould 2004), according to which

$$
\pi_{\mathrm{E}, \perp}^{\prime}=-\left(\pi_{\mathrm{E}, \perp}+\pi_{j, \perp}\right)
$$

where $\pi_{j, \perp}$ is the jerk parallax. From Equations (8) and (9) of Park et al. (2004), which make a number of simplifying approximations,

$$
\pi_{j, \perp}=-\frac{4}{3} \frac{\mathrm{yr}}{2 \pi t_{\mathrm{E}}} \frac{\sin \beta_{\text {ecliptic }}}{\left(\cos ^{2} \psi \sin ^{2} \beta_{\text {ecliptic }}+\sin ^{2} \psi\right)^{3 / 2}} \rightarrow-0.05,
$$

where $\psi \rightarrow 57^{\circ}$ is the ecliptic-latitude offset between the antiSun and the event at $t_{0}$. Because Earth's projected acceleration is within 0.2 of due west at the peak of the event, $\pi_{\mathrm{E}, \perp} \simeq \pi_{\mathrm{E}, N}$. (Note that because the $\left(\pi_{\mathrm{E}, \|}, \pi_{\mathrm{E}, \perp}\right)$ system is right-handed, $\pi_{\mathrm{E}, \perp} \sim \pi_{\mathrm{E}, N}$ for the first several months of the microlensing season and $\pi_{\mathrm{E}, \perp} \sim-\pi_{\mathrm{E}, N}$ during the last several months. See, e.g., Figure 3 from Park et al. 2004.) Therefore, from the fact that one $\left(s<0, u_{0}<0\right)$ solution has $\pi_{\mathrm{E}, N}=1.39$, the jerkparallax formalism predicts that the other should have $\pi_{\mathrm{E}, N}^{\prime}=-\left(\pi_{\mathrm{E}, N}+\pi_{j, \perp}\right)=-1.34$. The actual value $(-1.12)$ is in reasonable agreement, given the error bars. The situation is qualitatively similar for the other three pairs of solutions. We note that the jerk-parallax degeneracy has never previously been investigated in the context of planetary microlensing events. The caustic geometries of these eight solutions are shown in Figure 2, and their parameters are given in Tables 2 and 3 .

The first point to note about these eight solutions is that they have similar $\chi^{2}$, which means that all must be considered as viable. To facilitate the further discussion of these eight solutions, we label them by $C_{ \pm \pm}$or $W_{ \pm \pm}$. The letter stands for 


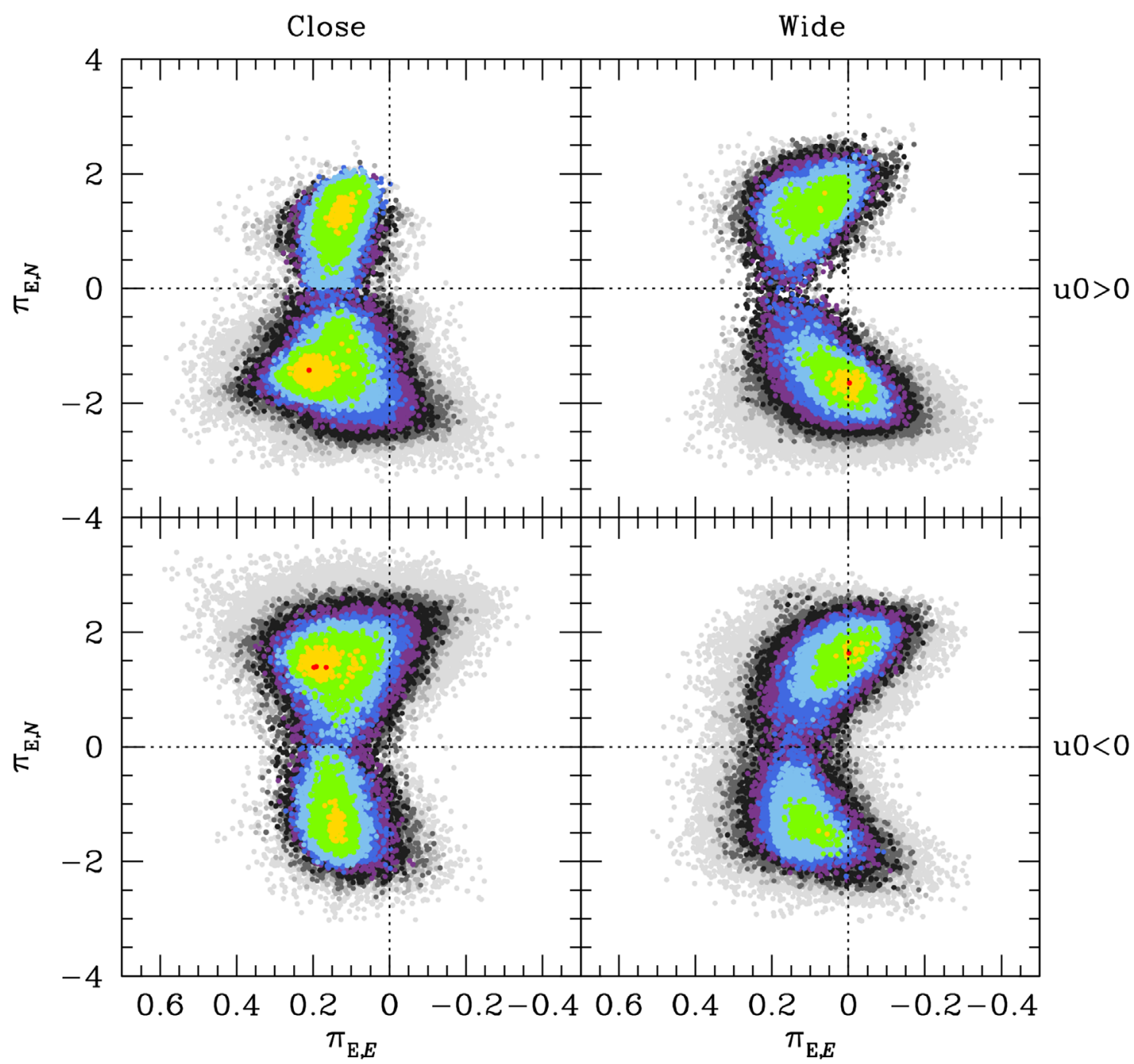

Figure 3. Scatter plots from the MCMC of the four solutions (close, wide) $\times\left(u_{0}<0, u_{0}>0\right)$ of KMT-2018-BLG-1990. Each shows two distinct minima, which correspond to the jerk-parallax degeneracy. Models with $\Delta \chi^{2}<\left(1^{2}, 2^{2}, \ldots, 10^{2}\right)$ are shown in red, gold, green, cyan, blue, magenta, black, dark gray, medium gray, and light gray, respectively. Note that the abscissa and ordinate axis scales are incommensurate.

close or wide (for $s<1$ or $s>1$ ), while the two subscripts refer to the signs of $u_{0}$ and $\pi_{\mathrm{E}, N}$, respectively.

Second, we note that six of the eight solutions have very comparable values of $q$ : the close solutions have $q \simeq 3.6 \times$ $10^{-3}$, while the $W_{+-}$and $W_{-+}$solutions have $q \simeq 4.2 \times 10^{-3}$. On the other hand, the solutions $W_{++}$and $W_{--}$have significantly higher $q \simeq 5.6 \times 10^{-3}$. Note that these two solutions have $\Delta \chi^{2} \sim 4$ relative to the best solution, and therefore this higher mass ratio will end up getting less weight in the final parameter estimates.

Third, all eight solutions have qualitatively comparable values of $\pi_{\mathrm{E}}$. That is, in all cases $\left|\pi_{\mathrm{E}, N}\right| \gg\left|\pi_{\mathrm{E}, E}\right|$ and in all cases $\left|\pi_{\mathrm{E}, N}\right|$ have comparable values. These facts together mean that $\pi_{\mathrm{E}} \simeq\left|\pi_{\mathrm{E}, N}\right|$ are similar for all four solutions. Because the errors on $\pi_{\mathrm{E}, N}$ are relatively large, it is important to assess whether these solutions are well localized. We display the $\pi_{\mathrm{E}}$ distributions in Figure 3, which shows that they are indeed well localized.

Fourth, we note that the remaining microlensing parameters are also comparable between solutions, with the exception of the orbital parameters. However, these are basically just nuisance parameters, which are very poorly constrained and are included only to avoid biasing the parallax parameters.

To illustrate the correlation between parallax and orbitalmotion parameters, we show in Figures 4 and 5 corner plots for these four parameters for the cases $C_{-+}$and $C_{++}$, respectively. For the case of $C_{-+}$, we see that there is strong correlation between $d \alpha / d t$ and $\pi_{\mathrm{E}, E}$, but not among other parameters. This solution favors a somewhat improbably high value of $d \alpha / d t$, albeit at $\lesssim 2 \sigma$. However, because $\pi_{\mathrm{E}, E}$ is both small compared to $\pi_{\mathrm{E}, N}$ and quite well constrained, this correlation has almost no impact on the physical implications.

For the case of $C_{++}$, we see that there are strong correlations between $\pi_{\mathrm{E}, N}$ and both orbital-motion parameters. Hence, if we had failed to include $d s / d t$ and $d \alpha / d t$ (i.e., implicitly assumed an unphysical static system), one sees that the fitted value of $\pi_{\mathrm{E}, N}$ would have been lower. However, when we carry out this exercise, we find that suppression of the orbital motion reduces $\pi_{\mathrm{E}, N}$ by less than $1 \sigma$.

Examination of Figure 1 shows that the source straddles the caustic boundary for only three points: (1) a KMTC point at the peak of the caustic entrance, (2) a KMTA point near the peak of the caustic exit, and (3) a KMTS point on the fall of the caustic 


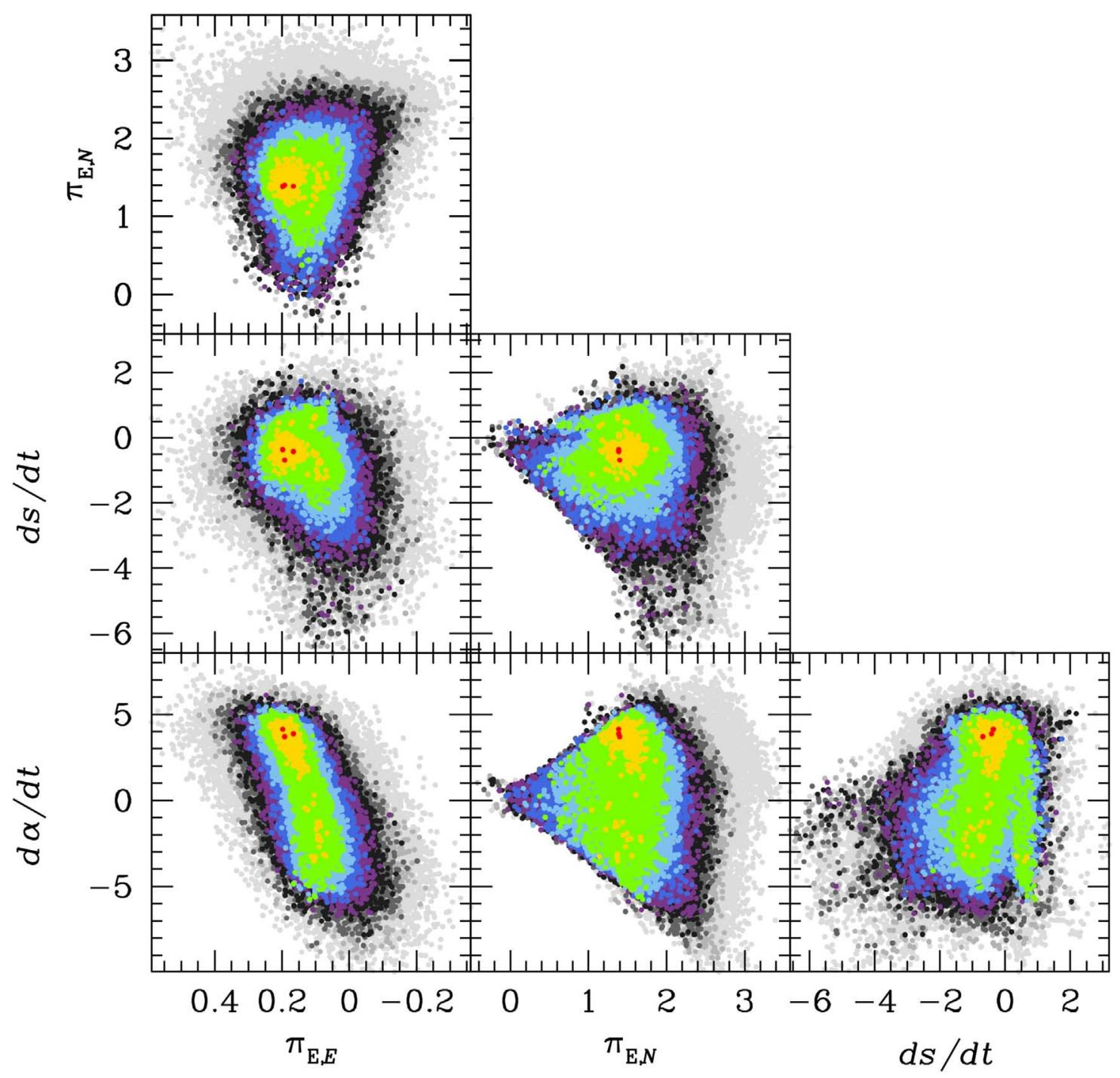

Figure 4. Corner plot showing the correlations between the two parallax parameters $\left(\pi_{\mathrm{E}, N}, \pi_{\mathrm{E}, E}\right)$ and the two orbital-motion parameters $(d s / d t, d \alpha / d t)$ based on the MCMC for the $C_{-+}$solution. The color have the same $\Delta \chi^{2}$ values relative to the local minimum as in Figure 3 . Note that some structure is induced on the ( $\left.\pi_{\mathrm{E}, N}, d s / d t\right)$ and $\left(\pi_{\mathrm{E}, N}, d \alpha / d t\right)$ panels by the constraint on the projected energy parameter $\beta<0.8$.

exit. The $\rho$ measurement rests entirely on these three points. Hence, we investigate the statistical consistency of the $\rho$ information coming from these three points, and we also examine the underlying images for systematics.

For the statistical investigation, we refit the $C_{-+}$solution (i.e., the solution with the best $\chi^{2}$ ) three times, with one of these three points removed in each fit. We find that each of these three truncated fits is consistent with the full fit, for which $10^{3} \times \rho=1.39 \pm 0.16$. For case (3; KMTS points removed), the $\rho$ distribution becomes bimodal, with solutions centered at $10^{3} \times \rho=1.21 \pm 0.09$ and at zero (i.e., no detected finitesource effects). This bimodality is analogous to the bimodality analyzed in detail by Chung et al. (2017) for the case of point lenses with very sparse coverage of finite-source effects. See their Figure 3. From the present perspective, the only relevant point is that one of the two solutions is consistent with the full solution. For the first two points, the corresponding values are $10^{3} \times \rho=1.38 \pm 0.25$ and $10^{3} \times \rho=1.37 \pm 0.20$, respectively. This statistical consistency of points from three different observatories indicates no evidence for systematic effects. It also shows that points (1) and (3) are contributing the most information. In particular, point (3) is required to establish that the finite source has been detected.

We also examine the individual images, including both the subtracted images (essentially consisting of an isolated difference star) and the residuals after subtraction of the model point-spread function (PSF). We find that for the KMTC point (with a FWHM of 4.64 pixels), the residuals after subtracting the model PSF are consistent with pure noise. However, for each of the KMTA and KMTS points (with a FWHM of 3.27 and 3.20 pixels), there is an anti-symmetric divot pattern in the residuals, with fractional amplitude of $\delta \simeq 7 \%$ and peak-trough separations of $\sim 3.0$ and $\sim 2.5$ pixels, respectively. This pattern is generically produced by an astrometric offset between the difference star and the PSF. See Figure 2 of Albrow et al. (2009). In the approximation of a Gaussian PSF, the peaktrough separation is equal to $\mathrm{FWMH} / \ln 4 \rightarrow 2.7$ pixels, in good agreement with the observed pattern. In this same approximation, the amplitude, $\delta$, is related to the fractional offset (relative to the FWHM), $a$, by $\delta=a \sqrt{\ln (256) / e}$, which implies $a=0.70 \delta \simeq 0.05$. This corresponds to 0.16 pixels or 


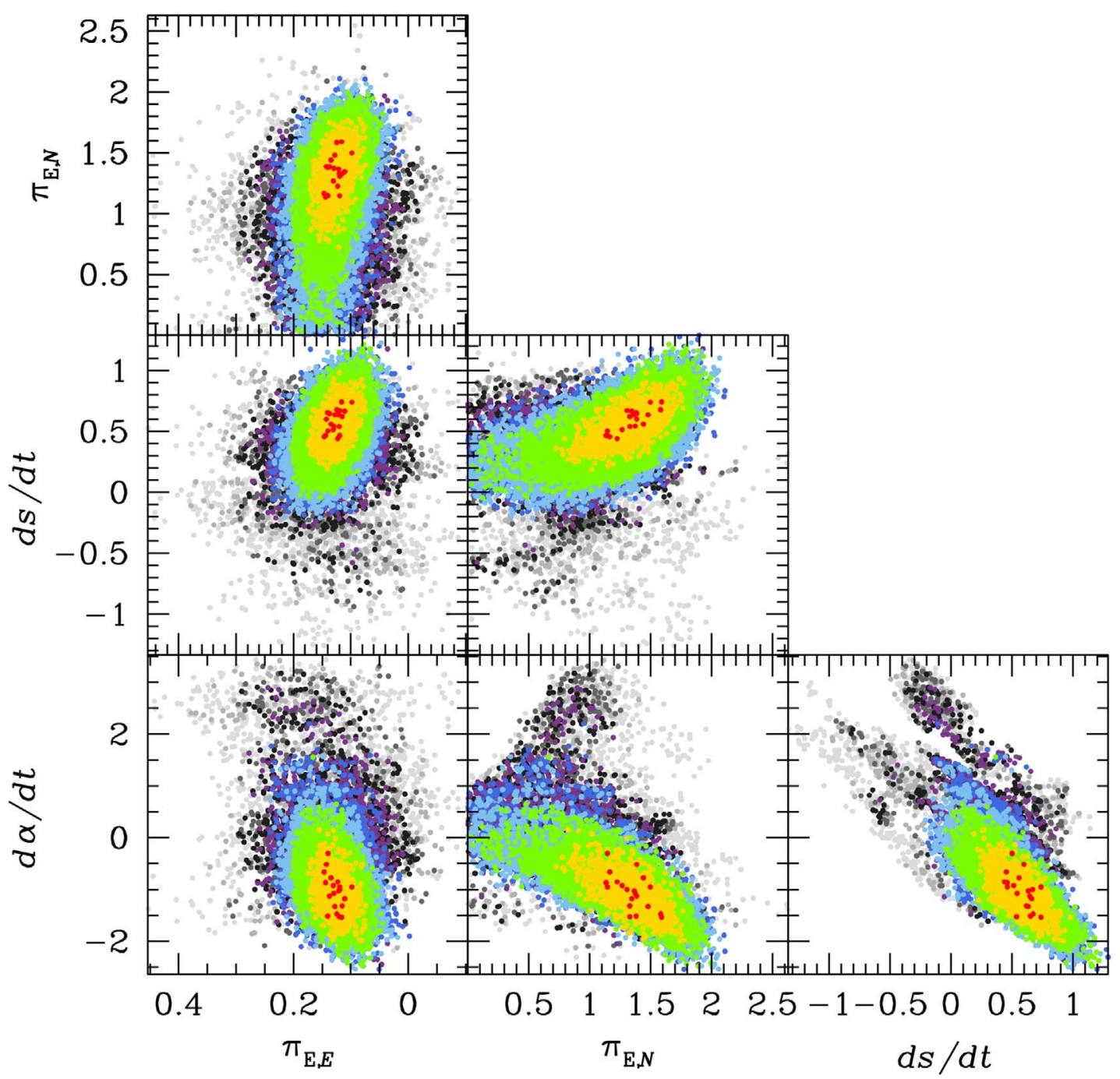

Figure 5. Similar to Figure 4, but for the case of the $C_{++}$solution.

about 65 mas. The fractional photometric error induced by this astrometric error can be estimated by interpolating $\sim 1 / 3$ of the way from the bright-source limit (dashed curve in Figure 1 of Albrow et al. 2009) to the faint-source limit (solid curve; analytically: $\left.(\ln 4) a^{2}\right)$. This yields a fractional error of 0.008 , which is a factor of three smaller than the photometric errors. We conclude that the $\rho$ measurement is robust within its quoted statistical errors.

\section{Physical Parameters}

Whenever $\theta_{\mathrm{E}}$ and $\pi_{\mathrm{E}}$ are well measured, one can always determine the relative parallax $\pi_{\text {rel }}$ and the lens mass $M$ from Equation (4), and so the lens distance $D_{L}=\mathrm{au} /\left(\theta_{\mathrm{E}} \pi_{\mathrm{E}}+\pi_{s}\right)$. In the present case, there are eight different solutions, but the bestfit values for each of these will lead to fairly similar values $M \sim 0.09 M_{\odot}, D_{L} \sim 0.8 \mathrm{kpc}$. This distance is unusually small because the phase space, which grows quadratically with distance, generally favors more distant lenses. Smaller values of the microlens parallax would, by Equation (4), give larger distances. Therefore, symmetric errors in $\pi_{\mathrm{E}}$ (which is essentially equal to $\left|\pi_{\mathrm{E}, N}\right|$ in the present case) should give rise to an asymmetric distribution in inferred distance around the value derived from the best fit. When the errors in $\pi_{\mathrm{E}}$ are small, this effect is likewise small and can be ignored. However, in the present case, both the variation in $\pi_{\mathrm{E}}$ between solutions and the statistical errors within solutions are fairly large. Hence, this effect must be accounted for. We will address this in Section 4.3. Before doing so, however, we must first evaluate $\theta_{*}$ (Section 4.1) and investigate the proper-motion distribution of the microlensed source (Section 4.2).

\subsection{Color-Magnitude Diagram}

The first step toward estimating physical parameters is to measure the source position on an instrumental CMD relative to the centroid of the red clump, which then permits one to estimate $\theta_{*}$ and thus $\theta_{\mathrm{E}}=\theta_{*} / \rho$. Note that in this method (Yoo et al. 2004), it is not necessary to calibrate the instrumental CMD, provided that the filters are themselves very nearly standard, which is the case for KMT $V$ and $I$. Next, we note that while the source color estimate does not depend on the microlensing model (and can often be derived by regression of $V$ on $I$ flux, without any model), the source magnitude does depend on the model. Therefore, for simplicity, we will explicitly derive results for the $C_{-+}$model and then present scaling relations for the rest. 


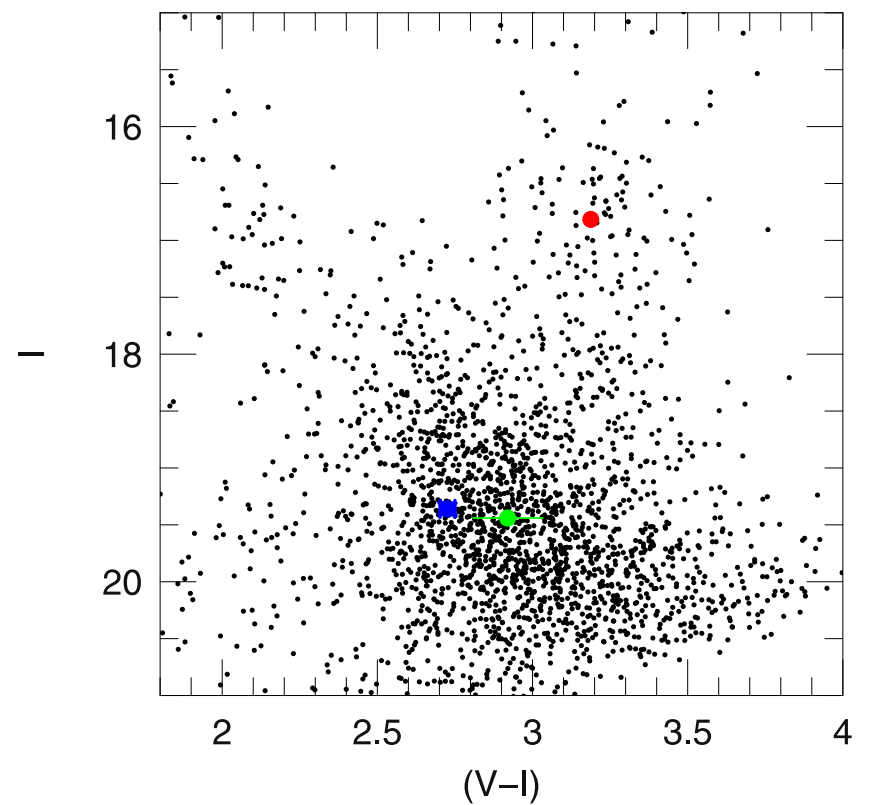

Figure 6. Instrumental CMD of stars within a $2^{\prime}$ square box centered on KMT2018-BLG-1990. The position of the microlensed source, the blended light, and the centroid of the red clump are shown as blue, green, and red circles, respectively. The values shown here are taken from the $C_{-+}$solution, but the other solutions would be virtually indistinguishable on this plot.

The CMD is shown in Figure 6, with the source and blend positions marked. The clump centroid is at $[(V-I), I]_{\mathrm{cl}}=$ $(3.19,16.82) \pm(0.02,0.08)$, whereas the source is at $[(V-I)$, $I_{s}=(2.73,19.36) \pm(0.02,0.05)$. The errors in the clump position are due to centroiding. The source color and error are derived from regression without reference to any model. The source magnitude error is set to 0.05 as representative of all solutions, which have very similar error bars. Hence, the offset from the clump is $\Delta[(V-I), I]=(-0.46,2.54) \pm(0.03$, $0.10)$. We adopt $[(V-I), I]_{\mathrm{cl}, 0}=(1.06,14.25)$ from Bensby et al. (2013) and Nataf et al. (2013), and so derive [(V-I), $I]_{s, 0}=(0.60,16.79) \pm(0.03,0.10)$. We have not included any error in the de-reddened position of the clump, but rather add an overall $5 \%$ error to $\theta_{*}$ due to all aspects of the method. We convert from $V / I$ to $V / K$ using the color-color relations of Bessell \& Brett (1988), and then use the color/surfacebrightness relation of Kervella et al. (2004) to derive

$$
\begin{aligned}
\theta_{*} & =1.23 \pm 0.09 \mu \mathrm{as} \\
\theta_{\mathrm{E}} & =0.88 \pm 0.11 \mathrm{mas} \\
\mu_{\mathrm{rel}} & =7.1 \pm 0.9 \mathrm{mas} \mathrm{yr}^{-1}
\end{aligned}
$$

where we have used $\rho=1.39 \pm 0.16$ from the $C_{-+}$solution, and where we have taken account of the anticorrelation between $f_{s}$ and $\rho$. For other solutions, one can simply scale $\theta_{*} / \theta_{*, C,-,+}=$ $\sqrt{f_{s} / 0.347}$ and $\theta_{\mathrm{E}} / \theta_{\mathrm{E}, C,-,+}=\sqrt{f_{s} / 0.347} /\left(\rho / 1.39 \times 10^{-3}\right)$.

\subsection{Source Proper Motion}

The source proper motion $\boldsymbol{\mu}_{s}$ is one of many inputs into a Bayesian estimate of the lens properties. For relatively bright sources, it is often possible to measure their proper motions, either from Gaia or from ground-based data. When this is not possible, one usually adopts an assumed distribution of source proper motions derived from a Galactic model. In the present case, the source is too faint and blended to measure its proper motion from ground data. In any case, the KMT time baseline would be too short for an accurate measurement. Moreover, the source does not appear in Gaia.

However, we can still use Gaia to measure the propermotion distribution of bulge stars, ${ }^{13}$ of which the microlensed source is very likely a member. We examine a Gaia CMD and on this basis select the 82 stars within $1^{\prime}$ and satisfying $G<18.6$ and $B_{p}-R_{p}>2.6$. We eliminate one outlier and derive (in the Sun frame)

$$
\begin{aligned}
& \left\langle\boldsymbol{\mu}_{\mathrm{bar}}(l, b)\right\rangle=(-5.4,0.1) \pm(0.3,0.2){\mathrm{mas} \mathrm{yr}^{-1}} \\
& \sigma\left(\boldsymbol{\mu}_{\mathrm{bar}}\right)=(2.9,2.2) \pm(0.2,0.2){\mathrm{mas} \mathrm{yr}^{-1}}^{-}
\end{aligned}
$$

\subsection{Bayesian Analysis}

Normally, one does not apply a Bayesian analysis when $\theta_{\mathrm{E}}$ and $\pi_{\mathrm{E}}$ are relatively well measured. Rather one would simply combine these measurements using Equation (4) to obtain $M$ and $\pi_{\text {rel }}$ (and so $D_{L}$ ), and propagate the errors in the measured quantities to the physical quantities. However, we argued at the beginning of Section 4 that in the present case a full Bayesian analysis was justified. Stating the argument differently, if the errors are small, then the frequentist and Bayesian approaches will yield nearly identical results, so there is no point employing the more cumbersome Bayesian formalism. And whether the errors can be considered small depends on the product of their absolute size and the gradient of the priors. In the present case, the errors should be considered large given the steepness of the gradient.

We draw random events from a Galactic model, as described in detail by Jung et al. (2018). The only exception is that we draw the source proper motions from a Gaussian distribution with the parameters that were derived from Gaia data in Section 4.2.

Each of the eight solutions is fed exactly the same ensemble of $10^{8}$ simulated events. For each solution, each simulated event is given a weight equal to the likelihood of its four inferred parameters $\left(t_{\mathrm{E}}, \theta_{\mathrm{E}}, \pi_{\mathrm{E}, N}, \pi_{\mathrm{E}, E}\right)$, given the error distributions of these quantities derived from the MCMC for that solution. Finally, we weight by $\exp \left(-\Delta \chi_{k}^{2} / 2\right)$, where $\Delta \chi_{k}^{2}$ is the difference in $\chi^{2}$ between the $k$ th solution and the best fit.

Hence, the $i$ th simulated event in the $k$ th solution is given a weight of

$$
w_{i, k}=\mathcal{L}_{i, k}\left(t_{\mathrm{E}}\right) \mathcal{L}_{i, k}\left(\theta_{\mathrm{E}}\right) \mathcal{L}_{i, k}\left(\pi_{\mathrm{E}}\right) \exp \left(-\Delta \chi_{k}^{2} / 2\right)
$$

where

$$
\mathcal{L}_{i, k}(X)=\frac{\exp \left[-\left(X_{i}-X_{k}\right)^{2} / 2 \sigma_{X_{k}}^{2}\right]}{2 \pi \sigma_{X_{k}}}, \quad X=t_{\mathrm{E}} \text { or } X=\theta_{\mathrm{E}},
$$

$$
\mathcal{L}_{i, k}\left(\pi_{\mathrm{E}}\right)=\frac{\exp \left[-\sum_{m, n=1}^{2} b_{m, n}^{k}\left(\pi_{\mathrm{E}, m, i}-\pi_{\mathrm{E}, m, k}\right)\left(\pi_{\mathrm{E}, n, i}-\pi_{\mathrm{E}, n, k}\right) / 2\right]}{(2 \pi)^{2} / \sqrt{\operatorname{det} b^{k}}},
$$

\footnotetext{
13 In fact, at this Galactic longitude $l=6.8$, the overdensity of stars on the sky is more usually referred to as the (near side of the) Galactic bar. Indeed, it is the rotation of the bar that causes the mean motion the bulge/bar stars to be

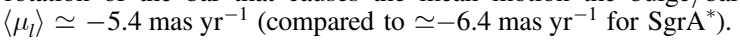



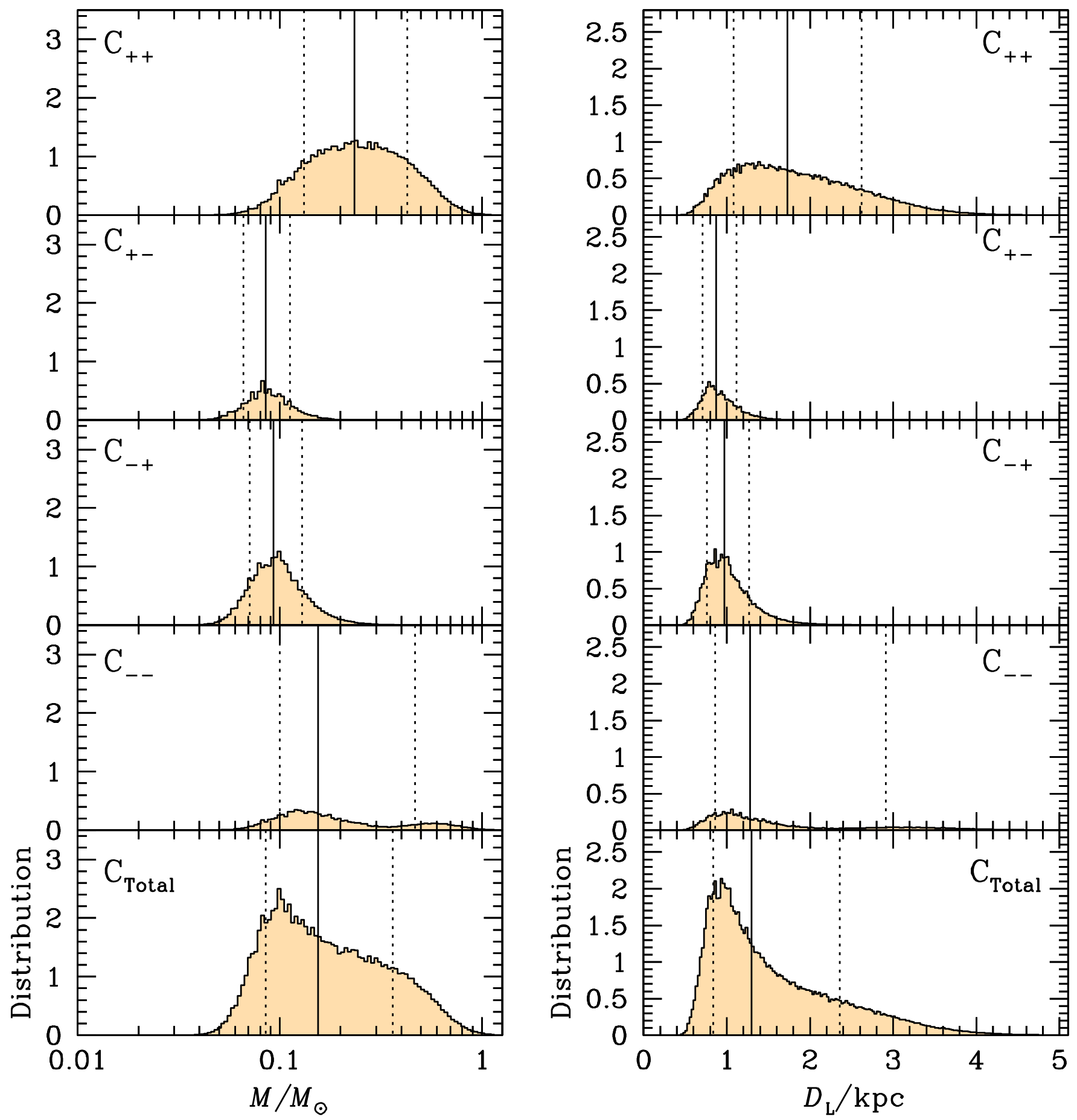

Figure 7. Histogram of the Bayesian posteriors of the lens mass and distance for each of the four of close solutions (top four rows) and the sum of all four solutions (bottom row). The total area in each histogram is proportional to the product of the weight derived from Bayesian analysis and $\exp \left(-\Delta \chi^{2} / 2\right)$ of the solution. The $C_{++}$ solution $\left(u_{0}>0, \pi_{\mathrm{E}, N}>0\right)$ dominates primarily because it is compatible with more distant lenses, which are more numerous in the Galactic model.

$b_{m, n}^{k}$ is the inverse covariance matrix of the $\pi_{\mathrm{E}}$ parallax vector in the $k$ th solution, and $(m, n)$ are dummy variables ranging over $(N, E)$.

The upper four sets of panels in Figures 7 and 8 show histograms for the lens mass and distance for each of the four close solutions and each of the four wide solutions, respectively. In each case, the relative area under the curve is the result of the weightings described by Equations (11)-(13). The relative weights can be evaluated from the last two columns of Table 4. In the bottom two panels of each figure, we show the combined histograms.
The first point to note is that, overall, the close solutions are significantly favored. This is mainly due to the fact that the $C_{++}$solution has the highest combined weight, which in turn reflects that it is compatible with disk lenses at relatively large distances (1-3 kpc), where the observation cone contains substantially more stars. The relatively broad range of lens distances of the dominant $C_{++}$solution (together with the well constrained $\left.\theta_{\mathrm{E}}=\sqrt{\kappa M \pi_{\text {rel }}}\right)$ likewise implies a relatively broad distribution of lens masses. In particular, for this solution the fractional error in $\pi_{\mathrm{E}, N}$ (and so, effectively, in $\pi_{\mathrm{E}}$ ) is about $29 \%$. 

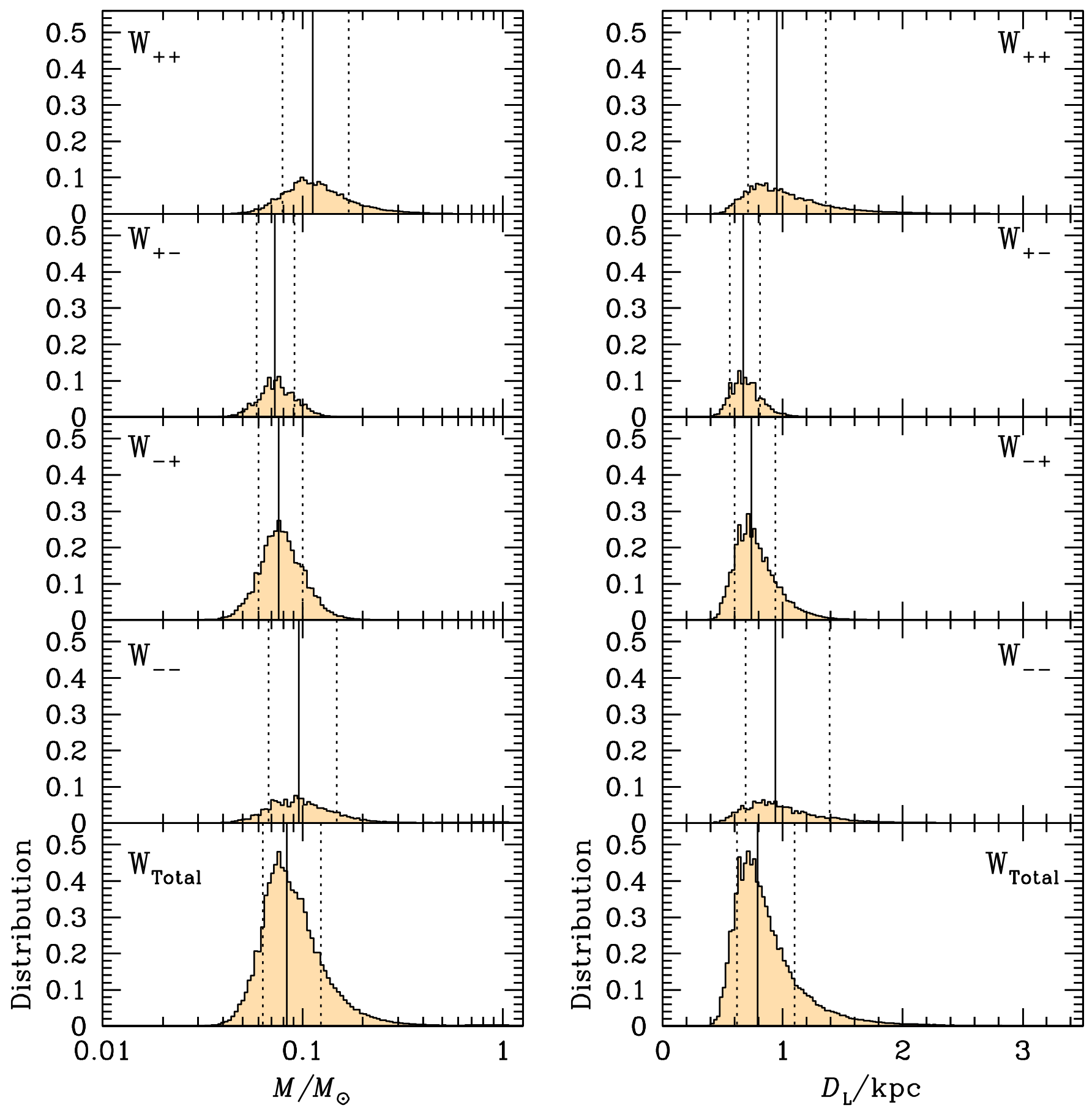

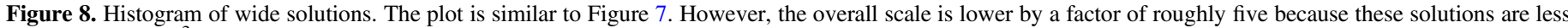
favored by both $\chi^{2}$ and the Galactic model.

As mentioned above, this is large relative to the gradient from the Galactic-model priors and so allows these priors to push the posterior lens distance much farther from the Sun than would be derived from the best-fit $\pi_{\mathrm{E}}$ from the light curve, i.e., $D_{L}=\mathrm{au} /\left(\theta_{\mathrm{E}} \pi_{\mathrm{E}}+\pi_{s}\right)$. The competition between the Galactic priors and the large-error parallax measurement then leads to fractional errors in the posterior mass estimate of $M=$ $0.235_{-0.104}^{+0.187} M_{\odot}=0.235 M_{\odot} \times 1_{-0.44}^{+0.80}$, which are substantially larger than the quadrature sum of the fractional errors in $\theta_{\mathrm{E}}$ and $\pi_{\mathrm{E}}$. We conclude that the lens is very likely to be a low-mass star, within a factor of two of $M=0.15 M_{\odot}$ at a distance of
0.8-2.3 kpc. See Figure 9. Because both the wide and close solutions have normalized separation $s$ quite close to unity, they do not substantially differ in their implications for the host-planet projected separation $a_{\perp} \equiv s \theta_{\mathrm{E}} D_{L}$ : the uncertainty in this quantity is dominated by the uncertain source distance. This uncertainty mainly factors out if we normalize this projected separation to the snow line, for which we adopt $a_{\text {snow }}=2.7 \mathrm{au}\left(M / M_{\odot}\right)$,

$$
\frac{a_{\perp}}{a_{\text {snow }}}=\frac{\kappa M_{\odot} s / 2.7}{\theta_{\mathrm{E}}+\pi_{s} / \pi_{\mathrm{E}}} \simeq \frac{(3 \mathrm{mas}) s}{\theta_{\mathrm{E}}+\pi_{s} / \pi_{\mathrm{E}}} .
$$


Table 4

Physical Properties

\begin{tabular}{|c|c|c|c|c|c|c|}
\hline \multirow[b]{2}{*}{ Models } & \multicolumn{4}{|c|}{ Physical Properties } & \multicolumn{2}{|c|}{ Relative Weights } \\
\hline & $M_{\text {host }}\left(M_{\odot}\right)$ & $M_{\text {planet }}\left(M_{J}\right)$ & $D_{L}(\mathrm{kpc})$ & $\overline{a_{\perp}(\mathrm{au})}$ & Gal. Mod. & $\chi^{2}$ \\
\hline$\overline{C_{++}}$ & $0.235_{-0.104}^{+0.187}$ & $0.923_{-0.409}^{+0.734}$ & $1.735_{-0.651}^{+0.889}$ & $1.521_{-0.555}^{+0.753}$ & 1.000 & 0.390 \\
\hline $\mathrm{C}_{-+}$ & $0.094_{-0.024}^{+0.036}$ & $0.348_{-0.087}^{+0.132}$ & $0.967_{-0.212}^{+0.308}$ & $0.763_{-0.145}^{+0.219}$ & 0.171 & 1.000 \\
\hline$C_{--}$ & $0.153_{-0.054}^{+0.311}$ & $0.605_{-0.215}^{+1.229}$ & $1.277_{-0.422}^{+1.629}$ & $1.083_{-0.315}^{+1.353}$ & 0.189 & 0.425 \\
\hline$C_{\text {Total }}$ & $0.153_{-0.068}^{+0.206}$ & $0.594_{-0.272}^{+0.814}$ & $1.296_{-0.452}^{+1.061}$ & $1.095_{-0.391}^{+0.953}$ & & \\
\hline$W_{+-}$ & $0.073_{-0.014}^{+0.018}$ & $0.318_{-0.058}^{+0.077}$ & $0.674_{-0.115}^{+0.139}$ & $0.657_{-0.075}^{+0.097}$ & 0.017 & 0.587 \\
\hline$W_{-+}$ & $0.076_{-0.016}^{+0.023}$ & $0.333_{-0.068}^{+0.101}$ & $0.742_{-0.138}^{+0.201}$ & $0.699_{-0.107}^{+0.145}$ & 0.039 & 0.785 \\
\hline$W_{--}$ & $0.095_{-0.027}^{+0.053}$ & $0.565_{-0.161}^{+0.316}$ & $0.937_{-0.243}^{+0.457}$ & $0.883_{-0.207}^{+0.379}$ & 0.078 & 0.152 \\
\hline$W_{\text {Total }}$ & $0.083_{-0.020}^{+0.039}$ & $0.398_{-0.112}^{+0.291}$ & $0.789_{-0.169}^{+0.316}$ & $0.751_{-0.137}^{+0.283}$ & ... & ... \\
\hline Total & $0.141_{-0.061}^{+0.202}$ & $0.565_{-0.249}^{+0.789}$ & $1.229_{-0.427}^{+1.063}$ & $1.039_{-0.351}^{+0.951}$ & $\ldots$ & $\ldots$ \\
\hline
\end{tabular}

Thus, for very nearby lenses, i.e., $\pi_{\mathrm{E}} \gg \pi_{s}, a_{\perp} / a_{\text {snow }} \rightarrow 3.4$, whereas for relatively distant lenses (within the framework of the Bayesian posteriors), $D_{L} \sim 3 \mathrm{kpc}, a_{\perp} / a_{\text {snow }} \rightarrow 2.0$. That is, the lens lies projected well outside the snow line over almost all of the posterior probability distribution.

\section{Discussion}

Despite relatively low-cadence observations, KMTNet obtained three caustic-crossing measurements of KMT-2018BLG-1990, which enabled a $\sim 14 \%$ measurement of the normalized source radius $\rho$ and hence good measurements of the Einstein radius $\theta_{\mathrm{E}}$ and the lens-source relative proper motion $\mu_{\text {rel }}$. When combined with a much weaker measurement of the microlens parallax $\pi_{\mathrm{E}}$ and incorporated into a Bayesian analysis, these measurements imply that the lens is very likely to be a low-mass $\mathrm{M}$ dwarf orbited by a gas giant planet in the Saturn-to-Jupiter mass range.

\subsection{Future Resolution}

The relatively high lens-source relative proper motion $\mu_{\text {rel }} \simeq 7$ mas yr $^{-1}$ implies that by 2028 (i.e., the roughly expected adaptive optics (AO) first light on next-generation $30 \mathrm{~m}$ telescopes), the lens and source will be separated by about 70 mas, which is easily enough to separately resolve them. At that time the remaining uncertainty in the lens mass and distance can be resolved. According to the Bayesian analysis presented in Section 4.3, there is a small chance that the lens is below the hydrogen burning limit, in which case it would most likely not be detected in such AO follow-up observations. Even in this case, however, it would be known to be a substellar object.

In principle, such a measurement could be made a few years earlier using $\mathrm{AO}$ on existing $10 \mathrm{~m}$ class telescopes. For example, Batista et al. (2015) used Keck to separately resolve the lens and source for OGLE-2005-BLG-169 when they were separated by only 61 mas, and Bennett et al. (2015) adequately resolved the source and lens using the Hubble Space Telescope (HST) when then were just 48 mas apart. However, in that case, the lens and source were comparably bright, whereas the source/lens flux ratio for KMT-2018-BLG-1990 could be very high.

\subsection{Probing Gas Giants Via Low-cadence Surveys}

This is the second KMT-only Jovian planet that has been detected in the $44 \mathrm{deg}^{2}$ of low-cadence $\Gamma \sim 0.4 \mathrm{hr}^{-1}$ KMTNet observations. This confirms the naive expectation, which we outlined in Section 1, that KMTNet's three-site survey at this cadence should be sensitive to such planets. Improving statistics on this population is crucial to our understanding of planet formation and early orbital evolution of planetary systems. Guided in part by the observed planet-mass distribution in the solar system and in part by theoretical consideration, it has long been predicted that the outer parts of extra-solar planetary systems should show a gap between Neptune-mass and Jovian-mass planets. Yet, the analysis of microlensing surveys (the only available means at present to probe cold planets that may lie in this gap) do not confirm these expectations. By obtaining a substantial sample of planets in this range, one can lay the basis for measuring their masses in subsequent AO follow-up observations, thus permitting a much stronger test of the gap hypothesis.

\subsection{Augmenting Low-cadence Surveys by Alerts and Follow-up}

The fact that the relatively low-cadence $\left(\Gamma=0.3 \mathrm{hr}^{-1}\right.$ and $\Gamma=0.4 \mathrm{hr}^{-1}$ ) KMTNet observations included three causticcrossing points is somewhat due to chance. It is these three observations that yielded a measurement of $\rho$ (and so $\theta_{\mathrm{E}}$ and $\mu_{\text {rel }}$ ), which enabled us to constrain the lens to be relatively nearby and of low mass. Without these measurements, the relatively long timescale $t_{\mathrm{E}} \simeq 45$ days would have led to a Bayesian mass estimate that was much greater. Thus, while planet detections that lack $\rho$ measurements are important, ${ }^{14}$ it is far better to obtain $\rho$ measurements if at all possible. In the present case, the source self crossing time is $t_{*} \simeq 0.06$ days. Thus, for approximately perpendicular caustic crossings, the total duration of two crossings (entrance and exit) is $4 t_{*} \sim 0.24$ days. Hence, even with $24 / 7$ coverage (no bad weather and no daylight when the target is at viewable airmass), one expects only $4 \Gamma t_{*} \simeq 2$ caustic-crossing points.

\footnotetext{
${ }^{14}$ Many planet detections in the future will lack $\rho$ measurements simply because they lack caustic crossings (Zhu et al. 2014). This was the case, in particular, for the previous KMT-only planet with $\Gamma=0.4 \mathrm{hr}^{-1}$ cadence, KMT-2016-BLG-1379 (Zang et al. 2018).
} 

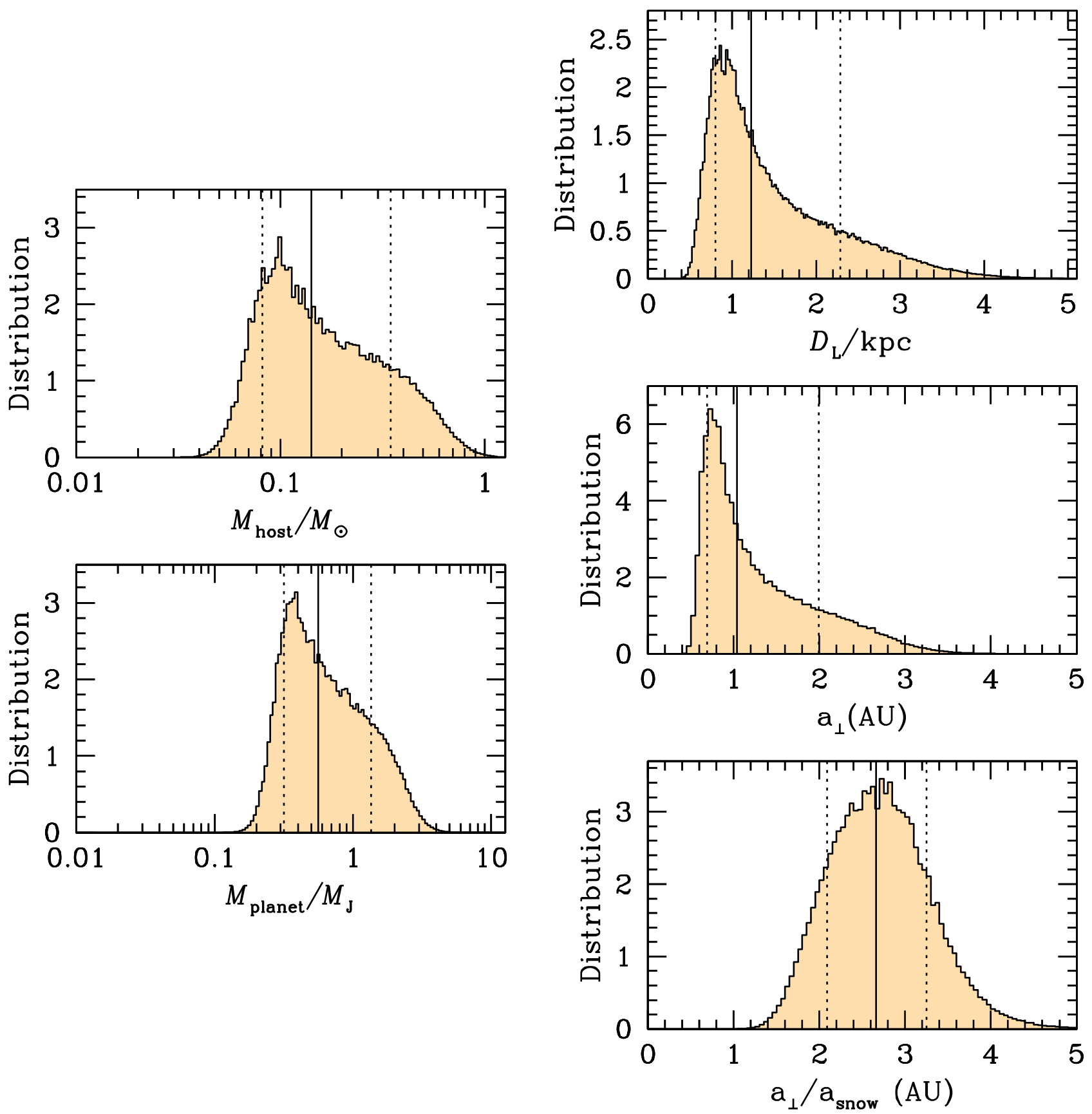

Figure 9. Posterior distributions of five physical quantities: host mass, planet mass, system distance, plant-host projected separation, and projected separation normalized to the snow line. Despite the parallax measurement, the allowed mass range is quite broad. Unless the lens proves to be substellar (for which there is only a small probability), its mass and distance will be decisively resolved at first light of next-generation $(30 \mathrm{~m})$ telescopes (or possibly before).

These considerations imply that follow-up observations, either triggered by real-time anomaly alerts or applied to realtime identification of relatively high-magnification events, could augment the science return of low-cadence microlensing planets considerably. Partly with this in mind, KMT has implemented real-time microlensing alerts ${ }^{15}$ (Kim et al. 2018c), which are announced each week day and are updated every three hours, 24/7 (except UT 03:00). If this system had been operational when KMT-2018-BLG-1990 was rising, then the event would have been alerted well before peak, and the caustic entrance at $\mathrm{HJD}^{\prime} \sim 8226.6$ would have been recognized within

\footnotetext{
15 http://kmtnet.kasi.re.kr/ulens/event/2019/
}

about $6 \mathrm{hr}$, and follow-up observations of the caustic exit $\sim 2.7$ days later could have been organized. In addition to guaranteeing a good measurement of $\rho$, a well-observed caustic exit might have broken some of the degeneracies among the eight solutions.

We thank the anonymous referee for an exceptionally helpful report. Work by A.G. was supported by AST-1516842 from the US NSF. I.G.S. and A.G. were supported by JPL grant 1500811. A.G. received support from the European Research Council under the European Unions Seventh Framework Programme (FP 7) ERC Grant Agreement No. [321035]. Work by C.H. was supported by the grant (2017R1A4A1015178) of 
National Research Foundation of Korea. This research has made use of the KMTNet system operated by the Korea Astronomy and Space Science Institute (KASI) and the data were obtained at three host sites of CTIO in Chile, SAAO in South Africa, and SSO in Australia.

\section{ORCID iDs}

Yoon-Hyun Ryu (i) https://orcid.org/0000-0001-9823-2907

Kyu-Ha Hwang (iD https://orcid.org/0000-0002-9241-4117

Michael D. Albrow (1D https://orcid.org/0000-0003-3316-4012

Sun-Ju Chung (1) https://orcid.org/0000-0001-6285-4528

Cheongho Han (iD https://orcid.org/0000-0002-2641-9964

Youn Kil Jung (ib https://orcid.org/0000-0002-0314-6000

In-Gu Shin (i) https://orcid.org/0000-0002-4355-9838

Yossi Shvartzvald (iD https://orcid.org/0000-0003-1525-5041

Jennifer C. Yee (i) https://orcid.org/0000-0001-9481-7123

Weicheng Zang (i) https://orcid.org/0000-0001-6000-3463

Richard W. Pogge (i) https://orcid.org/0000-0003-1435-3053

\section{References}

Alard, C., \& Lupton, R. H 1998, ApJ, 503, 325

Albrow, M. D. 2017, MichaelDAlbrow/pyDIA: Initial Release on Github, v1.0.0, Zenodo, doi:10.5281/zenodo. 268049

Albrow, M. D., Horne, K., Bramich, D. M., et al. 2009, MNRAS, 397, 2099

Batista, V., Beaulieu, J.-P., Bennett, D. P., et al. 2015, ApJ, 808, 170

Beaulieu, J.-P., Bennett, D. P., Fouqué, P., et al. 2006, Natur, 439, 437

Bennett, D. P., Bhattacharya, A., Anderson, J., et al. 2015, ApJ, 808, 169

Bensby, T., Yee, J. C., Feltzing, S., et al. 2013, A\&A, 549A, 147
Bessell, M. S., \& Brett, J. M. 1988, PASP, 100, 1134

Chung, S.-J., Zhu, W., \& Udalski, A. 2017, ApJ, 838, 154

Gould, A. 1992, ApJ, 392, 442

Gould, A. 1997, in Variables Stars and the Astrophysical Returns of the Microlensing Surveys, ed. R. Ferlet, J.-P. Maillard, \& B. Raban (Gif-surYvette: Editions Frontieres), 125

Gould, A. 2000, ApJ, 542, 785

Gould, A. 2004, ApJL, 606, 319

Gould, A., Carey, S., \& Yee, J. 2013, Spitzer Proposal ID, 10036

Gould, A., Carey, S., \& Yee, J. 2014, Spitzer Proposal ID, 11006

Gould, A., Carey, S., \& Yee, J. 2016, Spitzer Proposal ID, 13005

Gould, A., \& Loeb, A. 1992, ApJ, 396, 104

Gould, A., Yee, J., \& Carey, S. 2015a, Spitzer Proposal ID, 12013

Gould, A., Yee, J., \& Carey, S. 2015b, Spitzer Proposal ID, 12015

Gould, A., Yee, J., Carey, S., \& Shvartzvald, Y. 2018, Spitzer Proposal ID, 14012

Griest, K., \& Safizadeh, N. 1998, ApJ, 500, 37

Henderson, C. B., Gaudi, B. S., Han, C., et al. 2014, ApJ, 794, 52

Jung, Y. K., Udalski, A., \& Gould, A. 2018, AJ, 155, 219

Kervella, P., Thévenin, F., Di Folco, E., \& Ségransan, D. 2004, A\&A, 426, 297

Kim, D.-J., Kim, H.-W., Hwang, K.-H., et al. 2018a, AJ, 155, 76

Kim, H.-W., Hwang, K.-H., Kim, D.-J., et al. 2018b, AJ, submitted (arXiv:1804.03352)

Kim, H.-W., Hwang, K.-H., Shvartzvald, Y., et al. 2018c, ApJ, submitted (arXiv:1806.07545)

Kim, S.-L., Lee, C.-U., Park, B.-G., et al. 2016, JKAS, 49, 37

Nataf, D. M., Gould, A., Fouqué, P., et al. 2013, ApJ, 769, 88

Paczyński, B. 1986, ApJ, 304, 1

Park, B.-G., DePoy, D. L., Gaudi, B. S., et al. 2004, ApJ, 609, 166

Skowron, J., Udalski, A., Gould, A., et al. 2011, ApJ, 738, 87

Udalski, A., Jaroszyński, M., Paczyński, B., et al. 2005, ApJL, 628, L109

Woźniak, P. R. 2000, AcA, 50, 421

Yoo, J., DePoy, D. L., Gal-Yam, A., et al. 2004, ApJ, 603, 139

Zang, W., Hwang, K.-H., Kim, H.-W., et al. 2018, AJ, 156, 236

Zhu, W., Penny, M., Mao, S., Gould, A., \& Gendron, R. 2014, ApJ, 788, 73 